\title{
Transvenous Transseptal Concurrent Inoue Balloon Mitral and Aortic Valvuloplasty in an Octogenarian With Severe Aortic and Mitral Stenosis
}

\author{
Kae-Woei Liang, MD, PhD, ${ }^{\mathrm{a}, \mathrm{b}}$ and Yu-Cheng Chang, $\mathrm{MD}^{\mathrm{a}, \mathrm{c}}$ \\ ${ }^{a}$ Cardiovascular Center, Taichung Veterans General Hospital, Taichung, Taiwan \\ ${ }^{b}$ Department of Medicine, School of Medicine, National Yang Ming University, Taipei, Taiwan \\ ${ }^{c}$ Division of Cardiology, Department of Medicine, Asia University Hospital, Taichung, Taiwan
}

\begin{abstract}
An octogenarian was admitted because of severe aortic stenosis (AS) and mitral stenosis. He declined surgical double-valve replacement and underwent intracardiac echocardiography-guided transseptal puncture and concurrent Inoue (Toray, Tokyo, Japan) balloon aortic valvuloplasty (BAV) and balloon mitral valvuloplasty (BMV). Nine months later, he had worsening pulmonary edema and received a second session of Inoue BAV and BMV. Our case shows that concurrent transseptal Inoue BAV and BMV can treat severe AS and mitral stenosis with adequate safety and efficacy. However, the durability is limited in elderly persons with calcified AS. The procedure should be reserved for patients who refuse surgery.
\end{abstract}

Combined severe aortic stenosis (AS) and mitral stenosis (MS) is double obstruction of blood flow at the left side of the heart, resulting in a lower cardiac output and pulmonary edema. In the coexistence of MS and AS, the severity of valve stenosis can be underestimated because of the low-flow and low-gradient situation. ${ }^{1}$ The standard management should be combined surgical aortic and mitral valve replacement. ${ }^{1}$ Other options include transcatheter aortic valve implantation (TAVI) plus percutaneous transseptal Inoue (Toray, Tokyo, Japan) balloon mitral valvuloplasty (BMV), concurrent transvenous transseptal Inoue balloon aortic valvuloplasty (BAV) and $\mathrm{BMV},{ }^{2}$ or transvenous transseptal BMV and retrograde transarterial BAV.

Received for publication July 5, 2019. Accepted November 8, 2019.

Ethics Statement: The research reported has adhered to the relevant ethical guidelines.

Corresponding author: Dr Kae-Woei Liang, Cardiovascular Center, Taichung Veterans General Hospital, 1650 Taiwan Boulevard, Sec. 4, Taichung 40705, Taiwan. Tel.: +886-4-2359-2525 ext. 3121; fax: +886-4-23595046.

E-mail: ekwliang@gmail.com

See page 32 for disclosure information.

\section{RÉSUMÉ}

Un octogénaire a été admis en raison d'une sténose de l'aorte (SA) et d'une sténose mitrale (SM) graves. II a refusé de se soumettre à un double remplacement valvulaire par voie chirurgicale et a plutôt subi une intervention transseptale guidée par échocardiographie intracardiaque afin de réaliser en même temps une valvuloplastie aortique par ballonnet (VAB) et une valvuloplastie mitrale par ballonnet (VMB) selon la méthode d'Inoue (Toray, Tokyo, Japon). Neuf mois plus tard, il présentait un œdème pulmonaire allant en s'aggravant et a subi une deuxième intervention de VAB et de VMB d'Inoue. Le cas exposé montre qu'il est possible de traiter sans danger et efficacement une $S A$ et une SM graves par une VAB et une VMB d'Inoue réalisées par voie transseptale. La durabilité de l'intervention est toutefois limitée chez les personnes âgées présentant une SA calcifiée. L'intervention devrait être réservée aux patients qui refusent la chirurgie.

In decision making, fiscal constraint remained an issue for TAVI because many public insurances did not reimburse for expensive devices.

We present an octogenarian with severe MS and AS who strongly declined surgical double-valve replacement. Within 1.5 years, he underwent 2 rescue sessions of intracardiac echocardiography (ICE)-guided transseptal and concurrent transvenous transseptal BAV and BMV for relieving pulmonary edema.

\section{Case}

An 82-year-old man was referred to our hospital (Taichung Veterans General Hospital) because of combined severe AS and MS with worsening dyspnea and pulmonary edema. The baseline echocardiographic data revealed transaortic valve peak/mean pressure gradients of $71 / 33 \mathrm{~mm} \mathrm{Hg}$, transmitral valve peak/mean pressure gradients of $19 / 8 \mathrm{~mm} \mathrm{Hg}$, and normal left ventricular ejection fraction of 55\%. Hemodynamic data derived from cardiac catheterization showed a low cardiac output of $2.9 \mathrm{~L} / \mathrm{min}$ (by the Fick method) and systolic pulmonary arterial pressure of $81 \mathrm{~mm} \mathrm{Hg}$, which indicated a combined obstructive effect of 2 severe valvular stenoses. 


\section{Novel Teaching Points}

- Transvenous/transseptal concurrent Inoue BAV and BMV may be safe and effective in relieving heart failure in subjects with combined severe AS and MS.

- The durability is limited in senile calcified AS.

- Adding ICE guidance for transseptal procedure increases safety.

He strongly declined surgical double valve replacement after a full discussion with the cardiac surgeons. The heart team rejected him as a candidate for TAVI because of combined severe MS. The patient had pulmonary edema and persistent heart failure signs, so we decided to perform transvenous transseptal concurrent BAV and BMV on December 30, 2013. We followed the protocol proposed by Abdou et al., ${ }^{2}$ except that we modified the procedure using ICE to guide the transseptal puncture (Fig. 1). In brief, the transatrial septal puncture was performed using a Brockenbrough needle and Mullins sheath with ICE guidance (Figs. 1 and 2A). After transseptal puncture, the Mullins sheath was advanced from the right atrium (RA) to the left atrium (LA) (Fig. 2B) and positioned in the left ventricle (LV) (Fig. 2C). A Swan-Ganz balloon catheter was then advanced through the Mullins sheath to reach the LV and looped around the LV apex (Fig. 2C). A curved long wire $(260 \mathrm{~cm}$ long, 0.025 inch in diameter, J-curve) was placed inside the Swan-Ganz catheter, looping up from the LV apex to the outflow tract, crossing the aortic valve first to the ascending aorta and then to the descending aorta. The curved long wire was snared at the left external iliac artery (Fig. 2D) before being externalized outside the left femoral artery sheath, forming a stable right femoral vein, RA, LA, LV, aorta, left femoral artery wire loop, and rail. The Swan-Ganz catheter and Mullins sheath were then removed, leaving the guide wire with a wide loop in the LV. The stretched Inoue balloon catheter was advanced over the guide wire to the LA (Fig. 2E). The Inoue balloon catheter

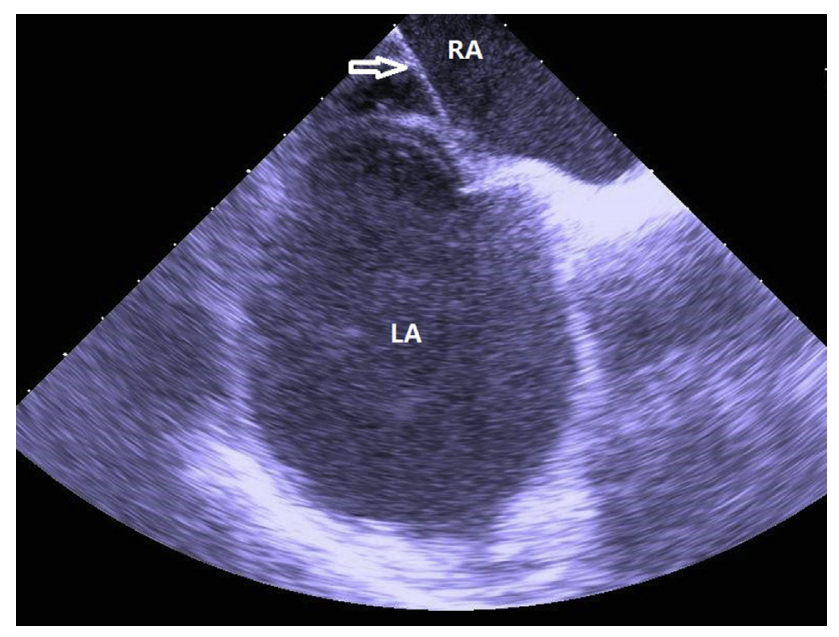

Figure 1. Intracardiac echocardiogram image showing tenting of transseptal needle on fossa ovalis. Arrow: transseptal needle. LA, left atrium; RA, right atrium. was de-stretched, and a stretch metal tube was withdrawn to leave its tip approximately $2 \mathrm{~cm}$ into the LA beyond atrial septum (Fig. 2F). The Inoue balloon was tracked via the 0.025-inch wire loop from the right femoral vein, RA, LA, and LV to the ascending aorta and was pulled back, saddled at the aortic annulus level, and inflated to $20 \mathrm{~mm}$ (BAV) (Fig. 2G). ${ }^{2}$ Afterward, the BMV was done with the same Inoue balloon inflated up to $24 \mathrm{~mm}$ at the mitral annulus level (Fig. $2 \mathrm{H}$ ). ${ }^{2}$ The transaortic valve mean pressure gradient decreased from 35 to $10 \mathrm{~mm} \mathrm{Hg}$, and the transmitral valve mean pressure gradient decreased from 15 to $5 \mathrm{~mm} \mathrm{Hg}$ after intervention. Pulmonary edema resolved in the following days, and the patient was discharged uneventfully. Nine months after the first intervention, he developed pulmonary edema and was again admitted to our hospital (Taichung Veterans General Hospital). The echocardiogram showed moderately severe AS with a transaortic valve mean pressure gradient of $23 \mathrm{~mm} \mathrm{Hg}$ and moderately severe MS with transmitral valve mean pressure gradient of $8 \mathrm{~mm} \mathrm{Hg}$. A repeated session of concurrent transvenous/transseptal Inoue BAV and BMV was performed successfully in September 2014. Pulmonary edema improved, and a few days later the patient was discharged. In January 2015, TAVI was scheduled, but the patient had tarry stool and coffee-grounds vomitus on the admission day and the plan was postponed. In February 2015, the patient fell accidentally, resulting in a bad head trauma with severe subdural hematoma. He died after neurosurgery.

\section{Discussion}

Antegrade transseptal concurrent Inoue balloon concurrent BAV and BMV has been proposed as an effective alternative treatment for subjects with combined AS and MS since 1998. ${ }^{3}$ The method has been modified in various versions over the past 20 years. The differences among those changes include the following: BMV first ${ }^{3}$ or BAV first, ${ }^{2}$ leaving the transvenous/transseptal wire in the descending aorta, ${ }^{3}$ or fixing it with a snare ${ }^{4}$ or snaring it and externalization ${ }^{2}$ outside the left femoral artery. We have modification of the procedure in adopting an ICE guidance in transseptal puncture, which could help visualize the atrial septum clearly and improve its safety. ${ }^{5}$ Other than this modification, we closely followed the protocol proposed by Abdou et al, ${ }^{2}$ with BAV first strategy, and snaring the transvenous/transseptal wire and externalizing it outside the left femoral artery to achieve better wire support and stability.

Previous reports showed a $0.5 \%$ systemic embolic event rate for transvenous BMV and a $1 \%$ stroke rate for retrograde transarterial BAV. There were also reports of a $1 \%$ event rate of aggravated severe mitral regurgitation requiring surgery after transvenous BMV and a $0.5 \%$ event rate of increasing 2 grades of aortic regurgitation (vs baseline) after transarterial BAV. The small-scale $(\mathrm{N}=14)$ study for transvenous, transseptal concurrent Inoue BAV/BMV reported no systemic thromboembolism or worsening regurgitation. ${ }^{2}$ To decrease thromboembolic complications, we suggested intraprocedure unfractionated heparin to maintain the activated clotting time over 250 seconds and postprocedure oral warfarin use for subjects with rheumatic heart disease and atrial fibrillation. Compared with transarterial retrograde BAV, transvenous/transseptal BAV has less entry site 


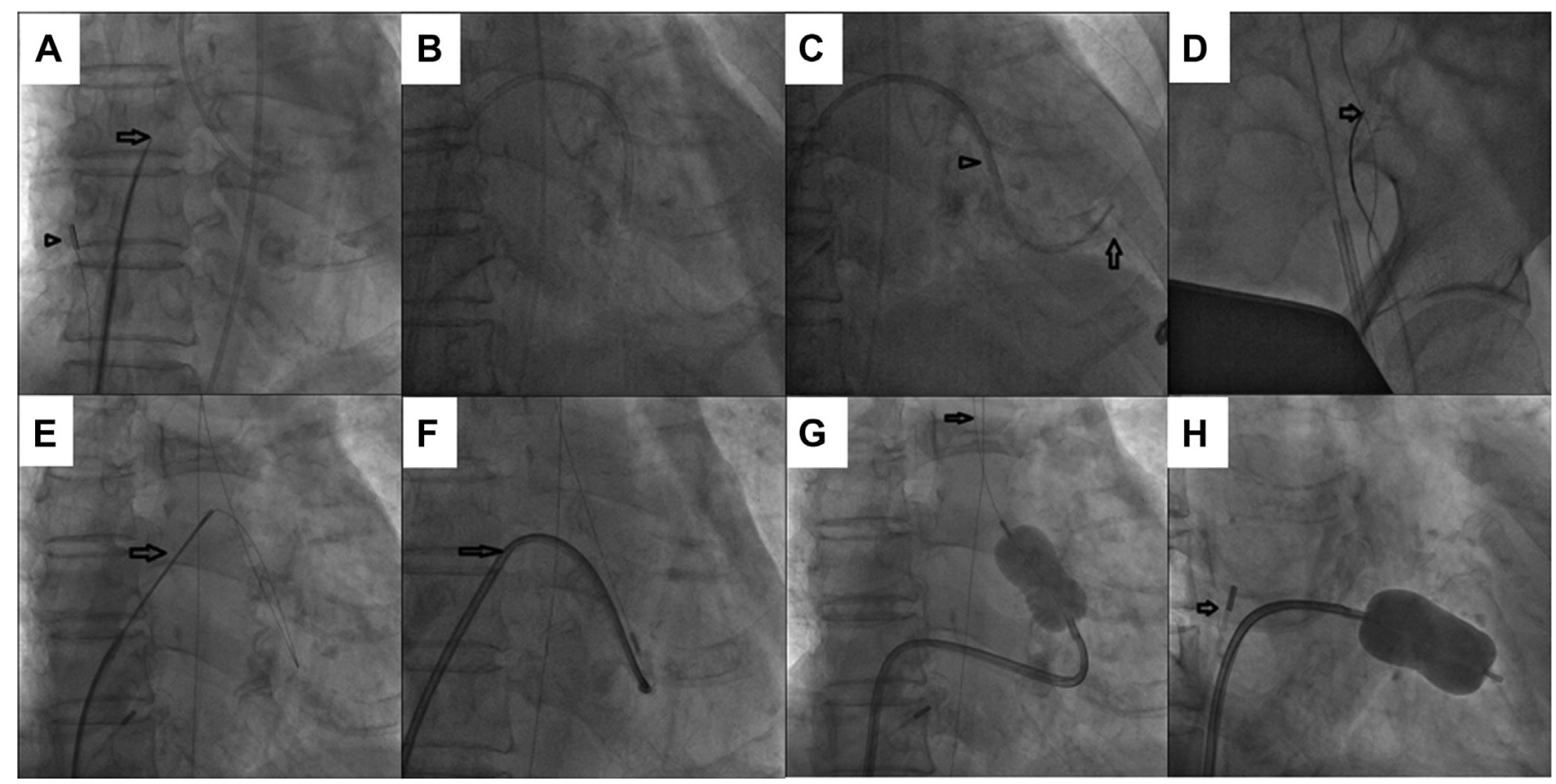

Figure 2. Step-by-step procedure sequences in transvenous transseptal concurrent Inoue (Toray, Tokyo, Japan) balloon mitral and aortic valvuloplasty. (A) The transseptal needle dilator (arrow) advances across the atrial septum into the left atrium (LA). Arrowhead: Intracardiac echocardiogram catheter. (B) After the transseptal procedure, the Mullins sheath was advanced to the LA. (C) The Swan-Ganz balloon catheter (arrow) was advanced to left ventricle (LV) through the Mullins sheath (arrowhead). With a slightly deep seating of the Mullins sheath in the LV, the balloon catheter was advanced to form an upward loop in the LV, directed toward the outflow tract and across the aortic valve to the ascending aorta. (D) A 260-cm, 0.025-inch wire was advanced inside the Swan-Ganz catheter from the ascending aorta to the abdominal aorta and then the iliac artery. The wire was externalized with a snare (arrow) at the left iliac artery for anchoring outside the left femoral arterial sheath. (E) The Swan-Ganz catheter and Mullins sheath were removed, leaving the guide wire with a loop in the LV. The stretched Inoue balloon catheter was advanced over the guide wire to the LA (arrow). (F) The Inoue balloon catheter was de-stretched, and the stretch metal tube was withdrawn to leave its tip (arrow) approximately $2 \mathrm{~cm}$ into the LA beyond the atrial septum. Thereafter, catheter manipulation was supported by the guide wire, anchored at both distal and proximal ends, as well as by stretch metal tube fixed across atrial septum (arrow). (G) The Inoue balloon was advanced to the ascending aorta, partially inflated, retracted to the saddle at the aortic annulus, and inflated up to $20 \mathrm{~mm}$. Arrow: The transvenous/transseptal wire (from the right femoral vein, RA, LA, LV, and ascending/descending aorta externalized at the left femoral artery) (H). The Inoue balloon was retracted to the mitral annulus level and inflated to $24 \mathrm{~mm}$. Arrow: Intracardiac echocardiogram catheter.

vascular injury and less LV wiring injury. Inoue balloon, compared with conventional balloons, also confers better aortic annulus sealing and dilatation effect for aortic valve stenosis. ${ }^{6,7}$

Regarding the efficacy and durability of transseptal concurrent BMV and BAV, past case series or registries usually reported better efficacy and durability in younger subjects with rheumatic AS and MS. ${ }^{8}$ Older adults with calcified degenerative AS had less favorable efficacy and treatment durability. For case selection, the concurrent transvenous transseptal BAV and BMV should be performed in younger subjects with rheumatic heart disease-related AS and MS with commissure fusion but without severe leaflet or annulus calcification and without preexisting more than moderate regurgitations. ${ }^{2}$ Our case is an elderly man aged 82 years with rheumatic MS but calcified AS, and thus had restenosis and clinical worsening 9 months after the first intervention.

\section{Conclusion}

Our case showed that transvenous/transseptal concurrent Inoue BAV and BMV may be safe and effective in relieving pulmonary edema and heart failure in subjects with combined severe AS and MS. However, the durability is limited in elderly patients with calcified aortic valves. The procedure should be reserved only for those who are unable to tolerate standard surgical double-valve replacement.

\section{Funding Sources}

The authors have no funding sources to declare.

\section{Disclosures}

The authors have no conflicts of interest to disclose.

\section{References}

1. Nishimura RA, Otto CM, Bonow RO, et al. 2014 AHA/ACC Guideline for the Management of Patients With Valvular Heart Disease: a report of the American College of Cardiology/American Heart Association Task Force on Practice Guidelines. Circulation 2014;129:e521-643.

2. Abdou SM, Chen YL, Wu CJ, Lau KW, Hung JS. Concurrent antegrade transseptal Inoue-balloon mitral and aortic valvuloplasty. Catheter Cardiovasc Interv 2013;82:E712-7. 
3. Bahl VK, Chandra S, Goswami KC. Combined mitral and aortic valvuloplasty by antegrade transseptal approach using Inoue balloon catheter. Int J Cardiol 1998;63:313-5.

4. Krishnan MN, Syamkumar MD, Sajeev CG, et al. Snare-assisted anterograde balloon mitral and aortic valvotomy using Inoue balloon catheter. Int J Cardiol 2007;114:e9-11.

5. Liang KW, Fu YC, Lee WL, et al. Intra-cardiac echocardiography guided trans-septal puncture in patients with dilated left atrium undergoing percutaneous transvenous mitral commissurotomy. Int J Cardiol 2007;117:418-21.
6. Eisenhauer AC, Hadjipetrou P, Piemonte TC. Balloon aortic valvuloplasty revisited: the role of the inoue balloon and transseptal antegrade approach. Catheter Cardiovasc Interv 2000;50:484-91.

7. Sakata Y, Syed Z, Salinger MH, Feldman T. Percutaneous balloon aortic valvuloplasty: antegrade transseptal vs. conventional retrograde transarterial approach. Catheter Cardiovasc Interv 2005;64:314-21.

8. Bahl VK, Chandra S, Goswami KC, Manchanda SC. Balloon aortic valvuloplasty in young adults by antegrade, transseptal approach using Inoue balloon. Cathet Cardiovasc Diagn 1998;44:297-301. 